\title{
Percutaneous Vertebroplasty in Multiple Myeloma: Prospective Long-Term Follow-Up in 106 Consecutive Patients
}

\author{
Giovanni Carlo Anselmetti • Antonio Manca • Filippo Montemurro • \\ Joshua Hirsch - Gabriele Chiara • Giovanni Grignani - Fabrizio Carnevale Schianca • \\ Antonio Capaldi · Delia Rota Scalabrini · Elena Sardo · Felicino Debernardi • \\ Gabriella Iussich • Daniele Regge
}

Received: 9 December 2010/ Accepted: 10 January 2011/Published online: 9 February 2011

(C) Springer Science+Business Media, LLC and the Cardiovascular and Interventional Radiological Society of Europe (CIRSE) 2011

\begin{abstract}
Purpose Percutaneous vertebroplasty (PV) is a minimally invasive procedure involving the injection of bone cement within a collapsed vertebral body. Although this procedure was demonstrated to be effective in osteoporosis and metastases, few studies have been reported in cases of multiple myeloma (MM). We prospectively evaluated the safety and efficacy of PV in the treatment of vertebral compression fractures (VCFs) resulting from MM.

Materials and Methods PV was performed in 106 consecutive MM patients who had back pain due to VCFs, the treatment of which had failed conservative therapies. Follow-up (28.2 \pm 12.1 months) was evaluated at 7 and
\end{abstract}

G. C. Anselmetti $(\bowtie)$ · A. Manca · G. Chiara · G. Iussich Interventional Radiology Unit, Institute for Cancer Research and Treatment, 10060 Candiolo, Turin, Italy e-mail: giovanni.anselmetti@ircc.it

A. Manca

e-mail: anto.manca@gmail.com

G. Chiara

e-mail: chiaragabriele@gmail.com

G. Iussich

e-mail: gabriellaiussich@yahoo.com

J. Hirsch

Interventional Neuroradiology, Massachusetts

General Hospital, Boston, MA, USA

e-mail: jahirschmd@yahoo.com

F. Montemurro - G. Grignani - F. Carnevale Schianca .

A. Capaldi · D. Rota Scalabrini

Oncology Unit, Institute for Cancer Research

and Treatment, 10060 Candiolo, Turin, Italy

e-mail: filippo.montemurro@ircc.it

G. Grignani

e-mail: giovanni.grignani@ircc.it
15 days as well as at $1,3,6,12,18$, and every 6 months after PV. Visual analog scale (VAS) pain score, opioid use, external brace support, and Oswestry Disability Index (ODI) score were recorded.

Results The median pretreatment VAS score of 9 (range $4-10)$ significantly $(P<0.001)$ decreased to 1 (range $0-9)$ after PV. Median pre-ODI values of $82 \%$ (range 36-89\%) significantly improved to $7 \%$ (range $0-82 \%)(P<0.001)$. Differences in pretreatment and posttreatment use of analgesic drug were statistically significant $(P<0.001)$. The majority of patients $(70$ of $81 ; 86 \%)$ did not use an external brace after PV $(P<0.001)$.

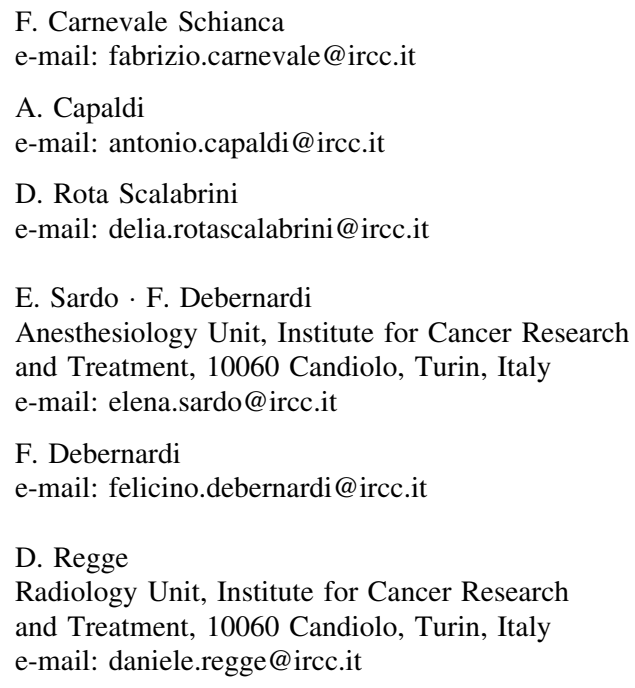


Conclusion PV is a safe, effective, and long-lasting procedure for the treatment of vertebral compression pain resulting from MM.

Keywords Vertebroplasty - Myeloma - Pain treatment . Interventional radiology $\cdot$ Quality of Life

\section{Introduction}

Multiple myeloma (MM) is a blood malignancy caused by transformation of plasma cells of B-lymphocyte origin that results in overproduction of monoclonal immunoglobulins. One of the distinctive features of MM is the production by malignant plasma cells of several cytokines that directly activate osteoclasts to remove compact bone, which leads to osteolytic bone lesions. In fact, bone involvement is present in 70-100\% of patients with MM and is associated with pain and skeletal-related complications, such as fractures and hypercalcemia. Despite being an incurable disease, considerable improvements in the prognosis of MM have been registered since the introduction of highdose chemotherapy with autologous hematopoietic support [1-4], newer biologically targeted agents, and the use of zoledronic acid [5]. Despite this recent increase in treatment options, a significant proportion of patients will eventually develop symptoms of bone progression during the course of the disease [6]. In fact, overall, bone metastases and their skeletal-related events are not controlled in approximately $20-40 \%$ of cancer patients [7-9], and the current therapeutic regimens leave $\leq 45 \%$ of patients with inadequate or undermanaged pain control $[10,11]$.

As survival rates in cancer patients improve, painful vertebral collapse becomes a more pervasive and clinically significant problem. Vertebral augmentation achieved through percutaneous vertebroplasty (PV) has become a mainstay in the treatment armamentarium. PV was first introduced in 1987 [12] for symptomatic vertebral angioma and then was used in osteoporotic fracture [13] and malignancy [14-17]. PV has gained world-wide acceptance as an effective minimally invasive treatment for back pain due to vertebral collapse not responding to conservative treatment. PV involves the injection of radio-opaque bone cement (polymethylmethacrylate [PMMA]) through a relatively thin needle (13-gauge in this series) within the collapsed vertebral body under image guidance and monolateral approach [18]. Kyphoplasty (KP) [19] uses an inflatable bone tamp generally introduced bilaterally through a larger cannula before PMMA injection to restore vertebral height and to decrease PMMA leakage. Preliminary retrospective studies have demonstrated the effectiveness of PV [16, 20] and KP [21, 22] in controlling pain and improving the quality of life of patients with painful MM of the spine. Few studies have prospectively analyzed the clinical outcomes achieved by PV [23] and KP [17] in relatively small series of patients. Both procedures are minimally invasive, can be performed with the patient under local anesthesia, seem to achieve the same clinical outcome [21] (i.e. pain relief), and have low complications rates.

Because the majority of MM patients are referred for vertebral augmentation after other treatments have failed, it may be necessary to treat patients with poor clinical conditions involving multiple vertebrae. It is the opinion of the lead author that in such cases, PV, using a thin-needle unipedicular approach, may be better indicated than KP, which more typically employs a bipedicular with a largergauge cannula.

PV is currently offered at the lead author's institution to patients with MM and symptoms related to spinal bone involvement that have become refractory to other medical treatments. The aim of this article is to report the long-term safety and efficacy of this procedure in a large, prospectively collected series of patients who have $\geq 1$ painful vertebral collapse secondary to MM.

\section{Materials and Methods}

\section{Study Design and Patient Population}

This study was designed as a single cohort using consecutive prospectively acquired data examining cement leakages, clinical complications, analgesic drugs requirements, external brace support, early and long-term pain management in patients with MM after PV as a treatment for osteolytic painful vertebral fractures that were poorly responsive to conventional therapies.

From 2002 to 2009, 625 consecutive vertebroplasty procedures were performed in 123 patients (58 women) with painful vertebral collapse resulting from MM during 145 sessions. 17 patients were lost to follow-up; data were prospectively collected in 106 patients (50 women) who underwent 528 consecutive vertebroplasty procedures during 124 sessions. The mean age was 70.1 years (range, 35-92 years). Indications for PV were painful vertebral compression fractures (VCFs) in association with myelomatous disease not responding to conventional therapies. Whereas, involvement of the posterior vertebral wall was not considered as an absolute contraindication; fractures with retropulsed tumoral tissue or bone fragments causing symptomatic spinal cord compression were not considered for PV. The mean duration of symptoms was 11.5 months (range, 4-23 months). Symptomatic levels were identified by correlating the clinical interview with magnetic resonance and computed tomography imaging in all patients. 
On average, 4.4 levels were treated per session (range from 1 to 13 ) from $\mathrm{C} 2$ to $\mathrm{S} 1$.

Patients were fully informed of potential treatment-related side complications and provided signed informed consent before each procedure in accordance with the Declaration of Helsinki. PV has been offered as a standard palliative treatment at our Institution since 2002. Outcomes data were collected in a prospectively maintained database and this analysis was approved by the Internal Review Board.

\section{Procedures}

PV was performed in most cases (87 of 106 patients; 82\%) using a C-arm angiographic unit (Advantx Tilt-C [GE Medical Systems, Milwaukee, MN] and Allura X-per CT [Philips, Best, The Netherlands]) with the patient in the prone position. Treatment of upper thoracic vertebrae (from T2 to T4) and the cervical spine was performed under combined fluoroscopic and computed tomography (CT) (LightSpeed16; GE Medical Systems) guidance according to the technique described by Gangi et al. [24]. PV was performed as previously described in the literature [18]. Bone biopsy, performed coaxially with a thru-cut needle (Magnum; Bard, New Jersey) before cement injection, confirmed MM diagnosis in all patients. Only local anesthesia was administrated in most cases (104 of 106 patients; 98.1\%), whereas general anesthesia (2 of 106 patients; $1.9 \%$ ) was performed during direct transoral approach (Fig. 1) to treat the second cervical vertebra. Postprocedural $\mathrm{CT}$ evaluation was performed in all patients to assess vertebral PMMA injection and to evaluate for cement leakage.

An average of $3 \pm 1.5 \mathrm{ml}$ PMMA was injected. In patients with multiple painful vertebral collapses, lowvolume multilevel vertebroplasty, consisting of injecting
$<3 \mathrm{ml}$ PMMA (average $1.5 \pm 0.5$ ) in each treated vertebra, was performed. Our logic was that this would minimize the risk of fat embolism and avoid depression of hematopoiesis $[25,26]$.

After the procedure, patients remain at strict bed rest for $2 \mathrm{~h}$ and are discharged when they are ready to ambulate, usually on the same procedural day.

\section{Assessment of Complications and Cement Leakage}

Clinical and procedural complications, as well cement leakages, were recorded prospectively at surgery. Postprocedural CT scans were performed in all patients to assess, more precisely than radiographs, local complications and cement leakages. If a venous cement leakage was encountered during the procedure, a postoperative CT scan of the lungs was also acquired to demonstrate pulmonary vein cement embolism. These data were recorded and correlated with any clinical symptoms during follow-up.

\section{Follow-Up and Clinical Outcome Evaluation}

A dedicated software database was developed to prospectively collect clinical data and technical information on treated patients. Demographic data, fracture locations, and treated levels were recorded at each session. Approach, equipment, cement type, and quantity were detailed for each treated vertebrae together with any technical or clinical complication.

Follow-up was completed by clinical interview ( 7 and 15 days after PV) and phone interview (at 1, 3, 6, 12, 18, and every 6 months). A clinical interview with radiological imaging was performed if the patients complained of new-onset back pain.
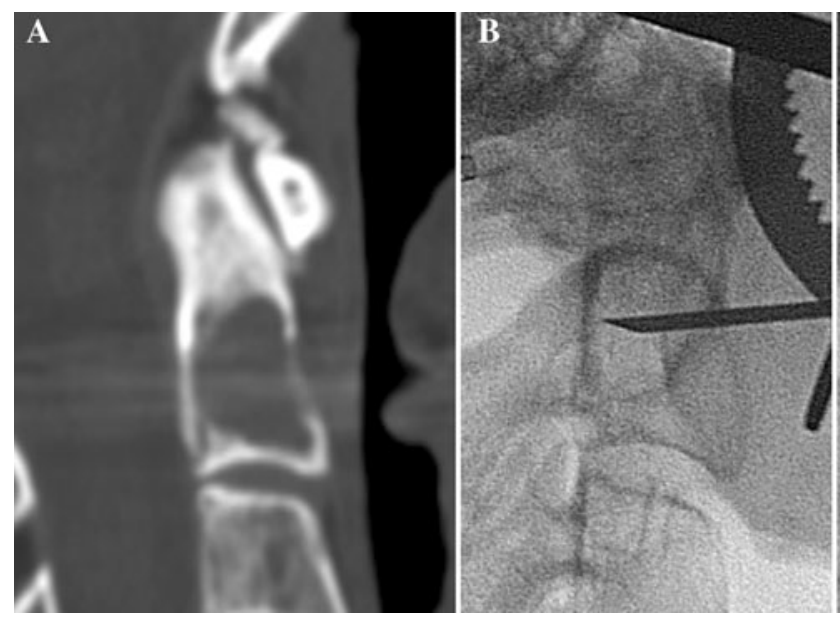

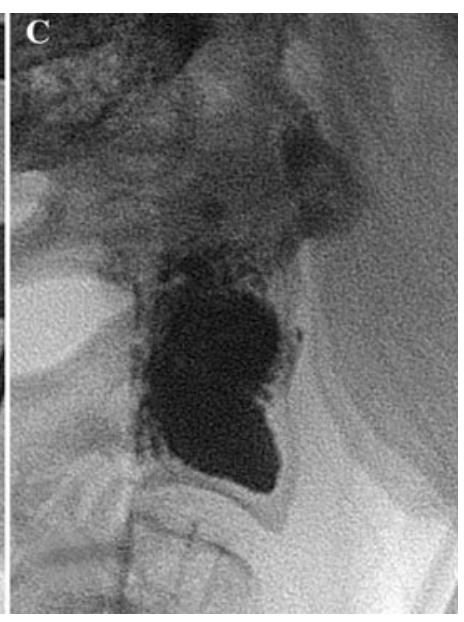

and radiotherapy. With a direct transoral approach (B), a 13-gauge needle is placed within the lesion. Bone consolidation with complete pain relief and without complication was achieved $(\mathbf{C})$ Fig. 1 Vertebroplasty of $\mathrm{C} 2$ with direct transoral approach. CT with
sagittal reconstruction (A) showed the persistence of a painful osteolytic lesion into the odontoid process of C2 after chemotherapy 
The Oswestry Disability Index (ODI) score, which represents the level of disability on a scale ranging from 0 (no disability) to $100 \%$ (bed bound), was obtained by means of a self-evaluation questionnaire that was completed by each patient just before PV (baseline) as well as at the time of the last interview (end point). Pain was evaluated with an 11-point pain intensity numerical rating visual analog scale (VAS) where 0 represents no pain and 10 represents the worst experienced pain. The raw change in the VAS score was computed by subtracting the baseline value from the end point for each patient. Farrar et al. [27], suggested that a decrease of two points in the raw change scores is clinically relevant. Analgesic drugs prescribed at baseline and at follow-up interviews were classified as none, nonsteroidal anti-inflammatory drugs (NSAIDs), oral narcotics, transdermal, or intravenous (IV) narcotic therapy with implanted pump.

\section{Statistical Analysis}

The outcome of interest was the change, with respect to pretreatment values, of the following variables at an average follow-up of $28.2 \pm 12.1$ months (range 12-72) after the procedure:

1. The ODI score was studied as a continuous variable with results rounded to the closest integer [28].

2. The VAS score was studied as both continuous and categorical ordinal variables.

3. The pattern of analgesic use, which was coded into four categories: $1=$ none, $2=$ NSAIDs, $3=$ oral opiates, and $4=$ transdermal/IV opiates was studied as both categorical nominal and categorical ordinal variables.

4. The use of an orthopedic brace was dichotomized into "yes" or "no."

Both the ODI and the VAS values (when treated as a continuous variable) were not normally distributed, even after logarithmic transformation. Therefore, for these variables, medians and ranges were chosen to summarize the data, and comparisons between pretreatment and posttreatment values were made by the Wilcoxon test. Categorical data, either nominal or ordinal, were studied by contingency tables. Pretreatment and posttreatment values were compared by the McNemar test (dichotomous variables), by the marginal homogeneity test (nominal variables with $>2$ levels), and by the sign test (ordinal variables with $>2$ levels).

The kinetics of pain improvement and the duration of benefit were studied by drawing Kaplan Meier curves of time to pain improvement and time to treatment failure. Time to pain improvement was calculated as the interval between the procedure and a VAS decrease of $\geq 2$ points evaluated at one of the follow-up visits. Time to treatment failure was calculated as the interval between the procedure and a VAS increase of $\geq 2$ points, the onset of new pain, the need for a new procedure, a serious adverse event requiring hospitalization, or death in the absence of disease progression. Significance was set $P<0.05$. Statistical analyses were performed by the SPSS version 17 statistical package (SPSS, Chicago, IL).

\section{Results}

PV was feasible in all 106 patients without any early major complications, such as death, symptomatic pulmonary embolism, and spinal or extraspinal tissues injuries.

Two out of 123 patients $(1.6 \%)$ had a delayed complication: one patient (excluded from long-term follow-up study) died from disseminated intravascular coagulopathy at 10 days after PV, and one patient (included in the study) developed a bacterial infection (Staphylococcus aureus) of the treated vertebra 30 days after the PV that required longterm IV antibiotic chemotherapy.

In five patients (4.7\%), a small PMMA embolism to the lungs was identified on CT scan. All of the cement pulmonary emboli were asymptomatic at all points, including immediately after the procedure and during long-term follow-up; no further treatment was necessary.

Sixteen patients $(15 \%)$ reported new-onset back pain during follow-up: X-ray examination and magnetic resonance imaging showed new vertebral collapse associated with overall disease progression. All new fractures were successfully treated at a second PV. Amongst the 16 patients, 2 had a further myelomatous vertebral fracture; thus, they underwent additional PV with apparent success.

Pain and Quality-of-Life Evaluation

Data regarding the variables of interest were available for the entire data set of 106 patients. The median pretreatment and posttreatment ODI values were $82 \%$ (range 36-89\%) and $7 \%(0-82 \%)$, respectively, and the difference was statistically significant $(P<0.001)$ (Fig. 2). All but 5 patients achieved an absolute decrease in ODI score, ranging from 7 to $88 \%$, after treatment. 27 patients (26\%) achieved an ODI score of $0 \%$ (no disability), and another 64 patients $(56 \%)$ achieved an ODI score of $<20 \%$ (minimal disability).

All of the patients had pretreatment VAS scores $\geq 4$ or higher. All but 5 patients achieved a decrease in VAS score as a result of treatment, with a median decrease of 7 points (range 0-10). The median pretreatment and posttreatment VAS values were 9 (range 4-10) and 1 (range 0-9), respectively, and the difference was statistically significant by both Wilcoxon test (considering VAS as a continuous variable $[P<0.001]$ ) and by signed test (considering VAS 


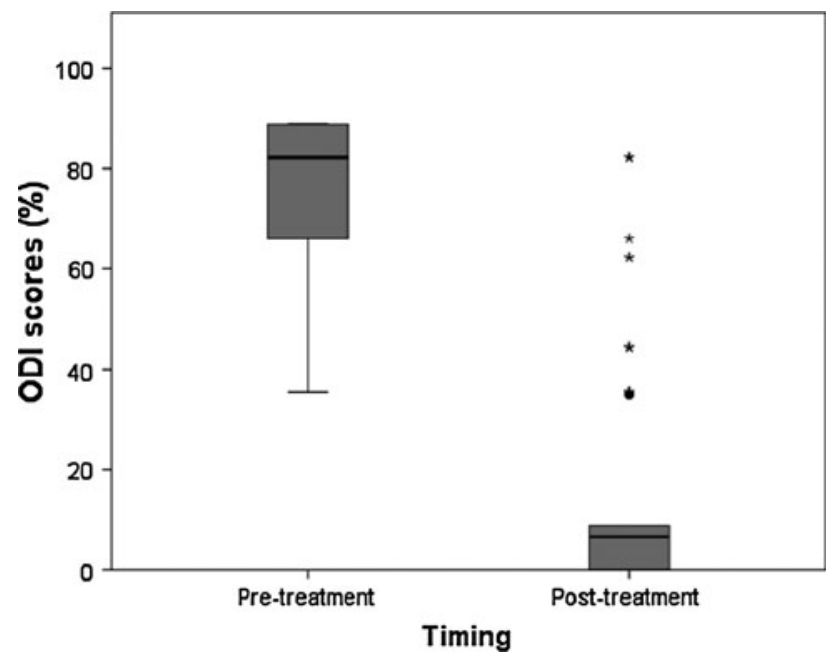

Fig. 2 Boxplots of ODI scores before and after treatment. The horizontal line in the middle of each box represents the median of the scores; the length of the box represents the interquartile range; and the whiskers represent smallest and highest values that are not outliers. Outliers (values between 1.5 and 3 box lengths) and extreme values (values that are more than three box lengths away) are indicated by an open circle and asterisk, respectively. Wilcoxon test $P<0.001$

as a categorical ordinal variable $[P<0.001])$. Notably, 101 patients (95\%) achieved a clinically relevant VAS decrease $(\geq 2)$; most (95 and 90\%) did so within $48 \mathrm{~h}$ of the procedure. 30 patients (28\%) achieved resolution of pain $(\mathrm{VAS}=0)($ Fig. 3). Figure 4 shows the time to treatment failure. At 12 months after the procedure, the rate of pain control was still close to $90 \%$.

\section{Use of Analgesic Drugs}

All of the patients were taking analgesic drugs before the procedure: $59(56 \%)$ of them were on opiates, either oral $(n=16[15 \%])$, transdermal $(n=40[38 \%])$, or IV $(n=3$ [3\%]). Table 1 lists the type and frequency of analgesic treatments used before and after treatment. Differences in

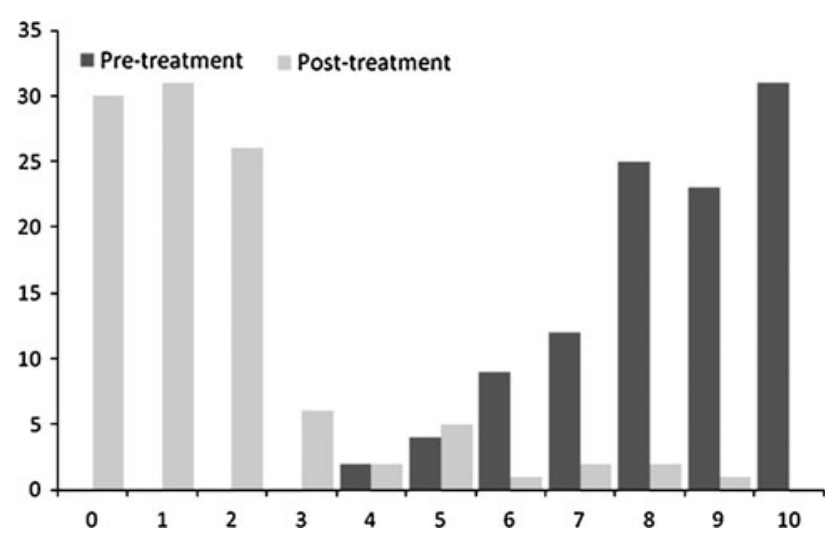

Fig. 3 Comparison of VAS scores before (dark bars) and after (light bars) treatment. Wilcoxon test $P<0.001$; signed test $P<0.001$

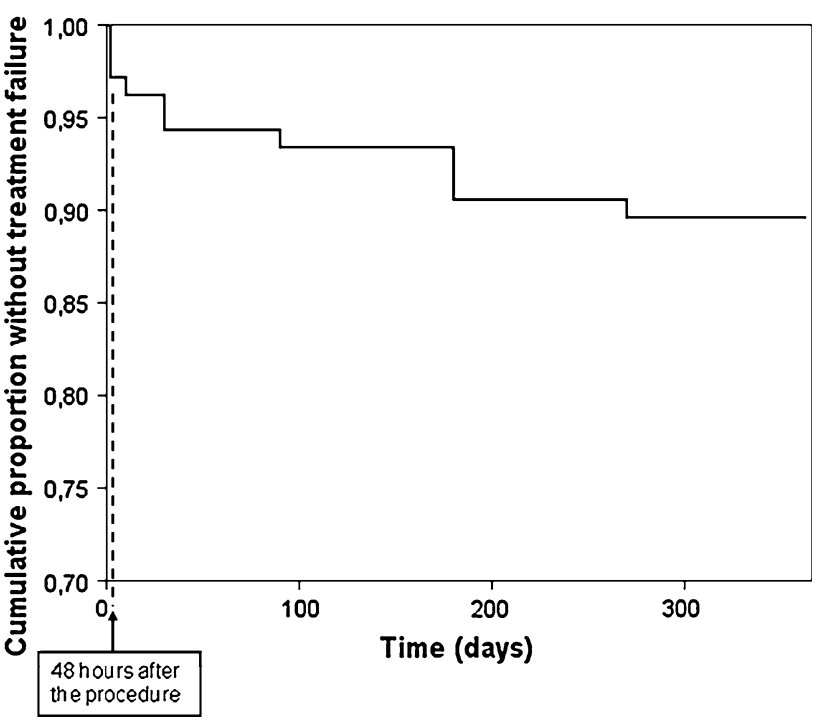

Fig. 4 Time to treatment failure

Table 1 Analgesic drug use before and after vertebroplasty

\begin{tabular}{lcc}
\hline $\begin{array}{l}\text { Type of analgesic } \\
\text { medication }\end{array}$ & $\begin{array}{l}\text { No. }(\%) \\
\text { before PV }\end{array}$ & $\begin{array}{l}\text { No. (\%) } \\
\text { after PV }\end{array}$ \\
\hline None & 0 & $54(51)$ \\
NSAIDs & $47(44)$ & $46(43)$ \\
Oral opiates & $16(15)$ & $3(3)$ \\
Transdermic/IV opiates & $43(41)$ & $3(3)$ \\
\hline
\end{tabular}

Marginal homogeneity test $P<0.001 ;$ sign test $P<0.001$

pretreatment and posttreatment frequencies in each analgesic drug category were statistically significant by both marginal homogeneity test $(P<0.001)$ and sign test $(P<0.001)$. All but 3 patients achieved a downshift in the category of analgesic drugs, with 54 patients $(51 \%)$ not requiring any analgesic.

\section{External Brace-Support Evaluation}

Before treatment, 81 patients wore an orthopedic brace (76\%). As a result of the procedure, 70 of these patients $(86 \%)$ no longer use the brace (Table $2, P<0.001$ ).

Table 2 Joined distribution of brace use before and after vertebroplasty

\begin{tabular}{llllr}
\hline & & \multicolumn{2}{l}{ Brace after PV } \\
\cline { 3 - 4 } & & No & Yes & \\
\hline Brace before PV & No & $25(100)$ & 0 & 25 \\
& Yes & $70(86)$ & $11(14)$ & 81 \\
& & 95 & 11 & 106 \\
\hline
\end{tabular}

Numbers in rows contain represent pretreatment frequencies, and numbers in columns represent posttreatment frequencies. Numbers in parentheses represent row percentages. McNemar test $P<0.001$ 


\section{Bone Cement Leakage}

Postprocedural CT detected a minimal bone cement extravasation in $121(22.9 \%)$ of the 528 treated vertebrae. Cross-sectional imaging with CT scans provides the most complete method of evaluating these leaks. These PMMA leaks were located in the perivertebral veins $(n=103$ $[85.1 \%])$, the disc space ( $n=7[5.9 \%])$, and the epidural vein $(n=11$ [9\%]). In 5 patients (4.8\%) PMMA leaked through the systemic veins into the lungs. All of these leaks were asymptomatic during the procedure, at postprocedural clinical observation, and at final follow-up.

\section{Discussion}

In this prospectively acquired database of a series of patients with MM and uncontrolled symptoms due osteolytic vertebral fractures, the use of PV proved to be safe and yielded long-lasting clinically meaningful benefits in the majority of treated patients. Baseline characteristics portray a group of patients with significant disease-related disability and pain despite the universal use of analgesic drugs, mostly consisting of opiates, in more than half of cases. Significant and durable decreases in disability scores, pain, and analgesic drug use suggest that PV should be considered a promising minimally invasive procedure in this patient setting.

For this analysis we selected patients who had at least 12 months of follow-up after the procedure because our main aim was to describe whether the benefits related to PV were durable. This selection did have the effect of excluding patients with more aggressive or late-stage disease, with a bias towards better outcomes that must be taken into consideration in interpreting our findings. Alternatively, with after-procedural follow-up ranging from 12 to 72 months (median 28 months), illustrates how crucial the optimal control of symptoms related to spinal metastases in patients whose survival can extend from several months to years after the diagnosis of this MM-related complication.

VCFs are frequent in MM, and may occur at the onset of the disease in $34-64 \%$ of the patient. Despite antitumor treatments and the use of bisphosphonates, the median risk for new fracture may be as high as $14 \%$ per year [29]. Severe pain and disability are usually treated with bed rest, bracing, radiotherapy, and analgesics, with limited benefit [30]. However, these treatments have been reported to fail in up to $45 \%$ of the cases, leaving patients with insufficient symptom control and significant disability [10, 11]. Furthermore, radiation therapy may be successful in allowing bone healing and stabilizing fractured vertebrae, but it usually takes weeks to months before the spinal column recovers its weight-bearing capabilities. In patients with poorly controlled symptoms of vertebral fractures, a vicious circle can occur where significant disability decreases compliance to anticancer treatment. In this case both PV and KP may be an attractive options for pain relief and faster recovery from fracture-relate disability. No comparisons are available between these two procedures in this clinical setting, but, in general, they are considered equivalent in terms of symptom control [31]. When multiple procedures are needed in the same patient, due to frequent presence of multiple collapsed vertebrae, the lead author believes that PV may be preferred to KP. Compared with KP, VP is possibly associated with greater incidence of bone cement leakage. In our series, this event occurred in approximately $23 \%$ of our patients, but it was usually minimal and with no associated symptoms during afterprocedural follow-up. Obviously, the frequency of bone cement leakage and of its potential consequences must be weighed against the overall benefit yielded by PV in this patient setting.

In conclusion, this study, in line with other reported retrospective series $[16,20]$, confirmed that $\mathrm{PV}$ is a safe ad effective treatment for of painful VCFs resulting from the progression of MM disease. PV produced pain relief and recovery from moderate to total disability, both of which were maintained during long-term follow-up. These results, especially recovery from disability with regain of mobility and decrease of analgesic drugs use, are likely to translate into improved patient compliance towards anticancer treatments.

Although prospective trials are needed to evaluate the worth of this procedure as an alternative or a complement to other supportive treatments, the results of our prospective case series strongly suggest that percutaneous vertebral augmentation is promising as an elective treatment in MM patients who suffer from uncontrolled pain and significant disability due to vertebral fractures.

Conflict of interest None.

\section{References}

1. Raab MS, Breitkreutz I, Anderson KC (2007) Targeted treatments to improve stem cell outcome: old and new drugs. Bone Marrow Transplant 40(12):1129-1137

2. Bjorkstrand B, Gahrton G (2007) High-dose treatment with autologous stem cell transplantation in multiple myeloma: past, present, and future. Semin Hematol 44(4):227-233

3. von Lilienfeld-Toal M, Hahn-Ast C, Furkert K et al (2008) A systematic review of phase II trials of thalidomide/dexamethasone combination therapy in patients with relapsed or refractory multiple myeloma. Eur J Haematol 81(4):247-252

4. Curran MP, McKeage K (2009) Bortezomib: a review of its use in patients with multiple myeloma. Drugs 69(7):859-888 
5. Musto P, Petrucci MT, Bringhen S et al (2008) A multicenter, randomized clinical trial comparing zoledronic acid versus observation in patients with asymptomatic myeloma. Cancer 113(7):1588-1595

6. Callander NS, Roodman GD (2001) Myeloma bone disease. Semin Hematol 38(3):276-285

7. Rosen LS, Gordon D, Tchekmedyian NS et al (2004) Long-term efficacy and safety of zoledronic acid in the treatment of skeletal metastases in patients with nonsmall cell lung carcinoma and other solid tumors: a randomized, phase III, double-blind, placebo-controlled trial. Cancer 100(12):2613-2621

8. Saad F, Gleason DM, Murray R et al (2004) Long-term efficacy of zoledronic acid for the prevention of skeletal complications in patients with metastatic hormone-refractory prostate cancer. J Natl Cancer Inst 96(11):879-882

9. Lipton A, Theriault RL, Hortobagyi GN et al (2000) Pamidronate prevents skeletal complications and is effective palliative treatment in women with breast carcinoma and osteolytic bone metastases: long term follow-up of two randomized, placebocontrolled trials. Cancer 88(5):1082-1090

10. de Wit R, van Dam F, Loonstra S et al (2001) The Amsterdam pain management index compared to eight frequently used outcome measures to evaluate the adequacy of pain treatment in cancer patients with chronic pain. Pain 91(3):339-349

11. Meuser T, Pietruck C, Radbruch L, Stute P, Lehmann KA, Grond S (2001) Symptoms during cancer pain treatment following WHO guidelines: a longitudinal follow-up study of symptom prevalence, severity and etiology. Pain 93(3):247-257

12. Galibert P, Deramond H, Rosat P, Le Gars D (1987) Preliminary note on the treatment of vertebral angioma by percutaneous acrylic vertebroplasty. Neurochirurgie 33(2):166-168

13. Watts NB, Harris ST, Genant HK (2001) Treatment of painful osteoporotic vertebral fractures with percutaneous vertebroplasty or kyphoplasty. Osteoporos Int 12(6):429-437

14. Anselmetti GC, Corrao G, Monica PD et al (2007) Pain relief following percutaneous vertebroplasty: results of a series of 283 consecutive patients treated in a single institution. Cardiovasc Intervent Radiol 30(3):441-447

15. Barragan-Campos HM, Vallee JN, Lo D et al (2006) Percutaneous vertebroplasty for spinal metastases: complications. Radiology 238(1):354-362

16. Masala S, Anselmetti GC, Marcia S, Massari F, Manca A, Simonetti G (2008) Percutaneous vertebroplasty in multiple myeloma vertebral involvement. J Spinal Disord Techn 21(5):344-348

17. Dudeney S, Lieberman IH, Reinhardt MK, Hussein M (2002) Kyphoplasty in the treatment of osteolytic vertebral compression fractures as a result of multiple myeloma. J Clin Oncol 20(9):2382-2387

18. Gangi A, Guth S, Imbert JP, Marin H, Dietemann JL (2003) Percutaneous vertebroplasty: indications, technique, and results. Radiographics 23(2):e10
19. Lieberman IH, Dudeney S, Reinhardt MK, Bell G (2001) Initial outcome and efficacy of "kyphoplasty" in the treatment of painful osteoporotic vertebral compression fractures. Spine (Phila Pa 1976) 26(14):1631-1638

20. McDonald RJ, Trout AT, Gray LA, Dispenzieri A, Thielen KR, Kallmes DF (2008) Vertebroplasty in multiple myeloma: outcomes in a large patient series. AJNR Am J Neuroradiol 29(4):642-648

21. Kose KC, Cebesoy O, Akan B, Altinel L, Dincer D, Yazar T (2006) Functional results of vertebral augmentation techniques in pathological vertebral fractures of myelomatous patients. J Natl Med Assoc 98(10):1654-1658

22. Astolfi S, Scaramuzzo L, Logroscino CA (2009) A minimally invasive surgical treatment possibility of osteolytic vertebral collapse in multiple myeloma. Eur Spine J 18(Suppl 1):115-121

23. Ramos L, de Las Heras JA, Sanchez S et al (2006) Medium-term results of percutaneous vertebroplasty in multiple myeloma. Eur J Haematol 77(1):7-13

24. Gangi A, Kastler BA, Dietemann JL (1994) Percutaneous vertebroplasty guided by a combination of CT and fluoroscopy. AJNR Am J Neuroradiol 15(1):83-86

25. Aebli N, Krebs J, Davis G, Walton M, Williams MJ, Theis JC (2002) Fat embolism and acute hypotension during vertebroplasty: an experimental study in sheep. Spine 27(5):460-466

26. Krebs J, Ferguson SJ, Hoerstrup SP, Goss BG, Haeberli A, Aebli $N$ (2008) Influence of bone marrow fat embolism on coagulation activation in an ovine model of vertebroplasty. J Bone Joint Surg Am 90(2):349-356

27. Farrar JT, Young JP Jr, LaMoreaux L, Werth JL, Poole RM (2001) Clinical importance of changes in chronic pain intensity measured on an 11-point numerical pain rating scale. Pain 94(2):149-158

28. Monticone M, Baiardi P, Ferrari S et al (2009) Development of the Italian version of the Oswestry disability index (ODI-I): a cross-cultural adaptation, reliability, and validity study. Spine (Phila Pa 1976) 34(19):2090-2095

29. Vogel MN, Weisel K, Maksimovic O et al (2009) Pathologic fractures in patients with multiple myeloma undergoing bisphosphonate therapy: incidence and correlation with course of disease. AJR Am J Roentgenol 193(3):656-661

30. Lecouvet F, Richard F, Vande Berg B et al (1997) Long-term effects of localized spinal radiation therapy on vertebral fractures and focal lesions appearance in patients with multiple myeloma. Br J Haematol 96(4):743-745

31. Hiwatashi A, Westesson PL, Yoshiura T et al (2009) Kyphoplasty and vertebroplasty produce the same degree of height restoration. AJNR Am J Neuroradiol 30(4):669-673 